\title{
PERFORMANCE ANALYSIS OF IMAGE COMPRESSION BASED ON FAST FRACTIONAL WAVELET TRANSFORM COMBINED WITH SPIHT FOR MEDICAL IMAGES
}

\author{
K. Ezhilarasan ${ }^{1}$, D. Jayadevappa ${ }^{2}$ and S. Pushpa Mala ${ }^{3}$ \\ ${ }^{1}$ Department of Electronics and Communication Engineering, Sambhram Institute of Technology, India \\ ${ }^{2}$ Department of Electronics and Instrumentation Engineering, JSS Academy of Technical Education, Bengaluru, India \\ ${ }^{3}$ Department of Electronics and Communication Engineering, Dayananda Sagar University, India
}

\begin{abstract}
Fast Fractional Wavelet Transform (FFWT) is an orthogonal linear transform called as decomposed signals in terms of chirps transform. This transform is used for signal and image compression and is based on Eigen value decomposition. In this paper, the performance analysis of image compression techniques based on the FFWT was discussed. FFWT is combined with the Set Partitioning in Hierarchical Tree (SPIHT) to achieve better compression ratio and biorthogonal filter banks for the analysis of compression performance with respect to subjective quality metrics. Further, the proposed work is compared with the various subject quantity parameters like PSNR and MSE.
\end{abstract}

\section{Keywords:}

Signal Compression, FFWT, SPIHT, Compression Ratio and Multiresolution Analysis

\section{INTRODUCTION}

Digital signal compression is a digital technique of representing a source of data with a fewer bits as compared to original data. This leads to the reduction in storage space, reduction in time for transmission without losing any significance details [1]. In literature there are various types of compression modalities and are grouped into three types namely, direct compression techniques, parameter extraction techniques and transformation techniques [2] [3]. In these types of data compression techniques, the redundancy of the signal will be reduced due to high decorrelation of coefficients.

It is noticed that, Fourier transform applied to obtain frequency domain signals finds the frequency parts present within the signal having zero time resolution. The fractional Fourier transform is viewed because the rotation of an indication through an angle $\alpha$ of Fourier transform. The fractional Fourier transform may be outlined generalizing the Fourier transform by rotating over an angle of $0.5 \pi$. Similar to fractional Fourier transform the fractional cosine and sine transforms [4]-[6] illustrates the energy density each in time and frequency plane having nonzero time frequency resolution. These transforms can be applied for optical signal process, sweep frequency filtering, linear time invariant filtering, pattern recognition, digital signal and image compression. In this paper, a compression technique adopting the Fast Fractional Wavelet Transform (FFWT) was proposed. Each wavelet component is differently scaled and band pass filtered in frequency domain. As we know, wavelet transform gives time resolution effectively than the frequency resolution. Hence, the wavelet transform ineffective in frequency domain where energy is not much significant in the frequency domain. Chirp like signals are universal in nature and manmade systems are examples of this forms. Hence, new innovative tools for signal analysis were introduced to investigate such type of signals. Examples for such tool are Fractional Wavelet Transform (FRWT) [7] [8], Fractional Fourier transforms (FRFT) [9] [10] and Short Time Fractional Fourier transforms (STFFT) [11]. Although the FRFT has a unique features, it does not provide local information of the signal. Also, due its limitations, the STFT will not provide time and fractional domain resolutions simultaneously with increased arbitrarily values.

The advantage of combining wavelet transform and the Fractional Resolution Fourier Transform (FRFT) is that, it is a linear transformation without cross term interference and is able to give multiresolution analysis by representing signals in the fractional domain. Hence, the fractional resolution Fourier transforms potentially helpful in the signal processing areas and this may give better prospects for future applications. In line with the fractional resolution Fourier Transform, Huang et al. [7] introduced the Fractional Wave Packet Transform (FRWPT) concept in 1998. But, this transform not attracted signal processing community much because of its physical interpretation and high computational time. Further, this transform is based on the fractional B-splines and is a scaled band pass filter in frequency plane and will not present signals in the fractional plane. But, still FRWPT will represent the conventional wavelet transform [10]. The main objective of this proposed technique is to provide a FFWT to compensate the drawbacks of wavelet transform and FRWPT. In the modified transform, every fractional wavelet element is scaled in a different manner so that perfect explicit physical interpretation can be achieved along with band pass filtering in the fractional domain. Therefore, this results lesser computational time as compared to the existing systems.

The special coding method that well suited for data to be coded having more zeros than nonzero is called SPIHT. The SPIHT algorithm proposed by Amir Said and Pearlman [12] is an advanced version of Embedded Zero Tree (EZW) algorithm. This algorithm possesses high Peak Signal Noise Ratio (PSNR) with better image quality and produces the complete embedded coding file. This method also provides an efficient error protection mechanism with fast coding and decoding process.

SPIHT is a coding method that sends coefficients first by transmitting MSBs of higher coefficients. This algorithm sorts out the within coefficients and transmits them along with the information which sorts bit by bit. Thus, bit rate for transmission can be accurately expressed so that only some of the MSBs of each coefficient are transmitted as like Discrete Fractional Wavelet Transform (DFRFT). Further, the SPIHT coding is free from mathematical calculations this is the most important advantage. In the proposed technique, significant nonzero 
coefficients are coded by applying SPIHT and bits were transmitted. Therefore, SPIHT results zero error. The combination of fractional Fourier transform and SPIHT results efficient compression ratio for the given image data.

This paper presents a scheme of transform coding for the application digital signal compression that uses Fast Fractional discrete Wavelet Transform (FFWT) as the transform domain. The Signal in a compact FFWT needs only a few FFWT coefficients and SPIHT encoding is used for binary coding of these coefficients. It is found that, there is an improvement in digital image compression as compared to the existing compression techniques which are available in the literature.

After a brief introduction to the proposed technique, related work is presented in section 2. In section 3, the proposed technique of digital image compression using DFWT with FFWT and SPIHT combination is discussed. The experimental results and its comparison with various bit rates were presented in section 4. The conclusion remarks are presented in section 5 .

\section{RELATED WORK}

Samil et al. [2] proposed an image compression technique using the fractional Fourier transform. This technique uses optimal filtering coefficients for multi-channel to minimize the MSE between the original matrix and its multi stage and multichannel approximation. Further, it is observed that, the Fractional Resolution Fourier transform (FRFT) in the time domain for image compression does not yield better results as they are applied for synthesis and fast implementation of the shift variant linear system. Vijaya et al. [13] proposed a signal compression technique using the discrete fractional wavelet transform and SPIHT. It is observed that the authors analyzed various elementary signals such as chirps, rectangular pulse and Gaussian signals.

All these signals were compressed with the SPIHT algorithm. It is also noticed that, the discrete fractional Fourier transform is suited for obtaining a better quality of reconstructed signal and percent RMS difference. Further, it does not require encoding the error signal and transmitting along with the encoded discrete fractional Fourier transform coefficients since the dynamic range of error signal is very small. Pushpa et al. [14] proposed a watermarking technique using the Fractional Wave Packet Transform (FRWPT) for medical images. In this paper, the FRWPT was applied for image compression. The results obtained with respect to the subjective metrics were quite good but the correlation coefficient was approximately equal to unity.

Pei et al. [15], presented a novel method for DFRFT computation at any angle is computed as the weighted summation of the DFRFT with specified angles, which are product of $2 \pi / N$ for all odd samples and $2 \pi / N+1$ for all even samples. Narayanan et al. [16] discussed and investigated about the FRFT theory, implementation an error analysis for various parameters for different applications such as DSP processor with ADSP-2192. Pei et al. [17] proposed the discrete fractional cosine transform and sine transform expected that significant progress will be made in the area of image processing and some more interesting application of FRFT in the area of control system and telecommunication system. Hsue et al. [18] presented the Rational ordered discrete fractional Fourier transform that investigated the periodicity and Eigen decomposition properties for all possible rational number orders. Candan et al. [19] developed the algorithm for discrete fractional Fourier transform to construct the Eigen value of decomposition of DFT matrix using Gauss Hermite Eigen function. Hyeh et al. [20] proposed the generalized angular decomposition for the DFRFT, behind the DFRFT decomposition, some other fractional signal transform also can be decomposed in to the weighted sums and the weighted sums coefficient are just equal to the DFT of the Eigen value. The number of decomposition angle is limited to the smallest odd integer that is not less than the length of the signal was proposed by [21].

\subsection{CONTINUOUS FFT}

It is observed that, the Fourier transform is linear transform, for a given function $x(n)$ the transform can be defined mathematically as,

$$
F\{x(n)\}=X(k)=\int_{-\infty}^{\infty} x(n) e^{-j 2 \pi k n} d n
$$

The FRFT $F^{\alpha}$ of order $a \in \mathbb{R}$ can be defined as,

$$
F^{\alpha}\{x(n)\}=X^{\alpha}(k)=\int_{-\infty}^{\infty} x(n) k^{\alpha}(n, k) d n
$$

where,

$F^{\alpha}$ represents the operator of fractional Fourier transform, and $k^{\alpha}(n, k)$ is the kernel function defined by

$$
\begin{aligned}
& k^{\alpha}(n, k)=\sqrt{\frac{1-j \cot \alpha}{2 \pi}} e^{j \cot \alpha\left(k^{2}+n^{2}\right)} \frac{e^{-j k n \operatorname{cosec} \alpha}}{2}{ }^{\alpha}(n, k)=x(x-n) \quad \text { for } \alpha \neq k \pi
\end{aligned}
$$

and

$$
x(k+n) \text { for }=(2 k-1) \pi
$$

The Chirps function plays a very significant role in the theory of FRFT.

\subsection{CHIRPS FUNCTION}

A chirps function sweeps the specific amount of frequency interval between $\left[\omega_{0}, \omega_{1}\right]$ with a regular time duration $\left[t_{0}, t_{1}\right]$. When the sweep rate is linear, then it can be represented in the form $e^{j \pi(\alpha x+\beta) x}$ and sweep rate as $\alpha$. The chirps function is defined as,

$$
F^{\alpha}(\delta(x-\beta))=k_{\alpha}(n, \beta)
$$

Further,

$$
\begin{aligned}
x^{2} \cot \alpha-2 x n \operatorname{cosec} \alpha+n^{2} \cot \alpha= & x^{2}(\cot \alpha-\operatorname{cosec} \alpha)+ \\
& (x-n)^{2} \operatorname{cosec} \alpha+ \\
& n^{2}(\cot \alpha-\operatorname{cosec} \alpha)
\end{aligned}
$$

For sweep rate of $\cot x$.

Thus, FRFT will be noticed by the application of a Chirpz convolution with $\operatorname{cosec} \alpha$ as the sweep rate between two Chirpz multiplications with a sweep rate $\cot x$. 


\subsection{DISCRETE TRANSFORM}

The $N \times N$ DFT can be expressed mathematically with the $N^{\text {th }}$ roots of unity as,

$$
X_{1}(k)=\sum_{n=0}^{N-1} x(n) e^{-\frac{j 2 \pi k}{N}}, \text { where } k=0,1, \ldots, N-1 .
$$

If $x(k, n)=\frac{W^{k n}}{\sqrt{N}}$, where $W=e^{\frac{j 2 \pi}{N}}$ is a phase factor. Then Eq.(7) can be written as,

$$
X_{1}(k)=\sum X_{1}(k, n) x(n) .
$$

Here, $X$ is the DFT matrix and satisfies $X^{4}=I$, where, $I$ is the identity matrix and it incorporates Eigen values,

$$
\{1,-i,-1, i\}=\left\{e^{\frac{j \pi k}{2}}, k=0,1, \ldots, N-1\right\}
$$

where, $N$ is the Orthonormal Eigenvectors that can be arranged as matrix $V$. Then its Eigen value decomposition is $X=V H V^{T}$ where, $H$ is a diagonal matrix.

The DFRFT forms a vector, and is given by,

$$
x=\{x(0), x(1), \ldots, x(N-1)\}^{T}
$$

In vector form, $x^{\alpha}=X^{\alpha} x$ with components defined by $X^{\alpha}$

$$
X^{\alpha}=x^{\alpha}(k)=\sum_{n=0}^{N-1} X^{\alpha}(k, n) x(n), k=0,1, \ldots, N-1
$$

where,

$$
X^{\alpha}=E H^{\alpha} E^{T}
$$

$X=V H V^{T}$ is the Eigen value decomposition of the DFT matrix, $H$ is the Sum of the circulant matrix $(A)$ and diagonal matrix $(B)$. The circulant matrix corresponds to the impulse response system and is expressed as,

$$
\begin{gathered}
h(n)=\delta(n+1)-2 \delta(n)+\delta(n-1) \\
B=X A X^{-1}
\end{gathered}
$$

The Eq.(13) is the diagonal matrix. Further, it can also be seen that, $X B X^{-1}=X^{2} A X^{2}=A$, Since, $h(n)$ is an even function then,

$$
X H X^{-1}=X(A+B) X^{-1}=B+A=H
$$

Finally, the DFRFT is represented as,

$$
\begin{gathered}
X^{\alpha}=\sum_{k=0}^{N-1} e^{-j k \alpha} V_{k} V_{k}^{T} \text { for } N=\text { odd } \\
X^{\alpha}=\sum_{k=0}^{N-2} e^{-j k \alpha} V_{k} V_{k}^{T}+e^{-j N \alpha} V_{N} V_{N}^{T} \text { for } N=\text { even }
\end{gathered}
$$

The output signal of DFRFT is computed as,

$$
\begin{gathered}
X^{\alpha}=\sum_{k=0}^{N-1} e^{-j k \alpha} V_{k} V_{k}^{T} x \text { for } N=\text { odd } \\
X^{\alpha}=\sum_{k=0}^{N-2} e^{-j k \alpha} V_{k} V_{k}^{T}+e^{-j N \alpha} V_{N} V_{N}^{T} x, \text { for } N=\text { even }
\end{gathered}
$$

\section{PROPOSED METHOD}

In this section, fractional wavelet transform, discrete fractional wavelet transform and the combination of FFWT combined with SPIHT were described to compute image compression analysis.

\subsection{FRACTIONAL WAVELET TRANSFORM}

The definition of 1-D wavelet transform is given by,

$$
W_{x}(a, b)=\int_{-\infty}^{\infty} x(n) \psi_{a, b}^{*}(t) d n
$$

where, $W_{x}(a, b)$ is the correlation between the input signal $x(n)$ and the mother wavelet function $\psi_{a, b}(t)$,

$\psi_{a, b}^{*}(t)$ is the mother wavelet function, it can be expressed as,

$$
\psi_{a, b}(t)=\frac{1}{\sqrt{a}} \psi\left(\frac{t-b}{a}\right)
$$

where, $b$ is the shifting parameter, $a$ is the scaling parameter and $\sqrt{a}$ normalization factor. This type of property was implemented perfectly in digital image compression techniques.

The fractional convolution of the FRFT for the functions $x(n)$ and $h(n)$ is defined as

$$
x(n) \odot \alpha h(n)=e^{\left(-\frac{j}{2}\right) n^{2} \cot \alpha}\left(x(n) e^{\left(\frac{j}{2}\right) n^{2} \cot \alpha} h(n)\right)
$$

where, $\bigodot_{\alpha}$ represents a fractional convolutional operator. Then the fractional convolution of the FRFT is given by

$$
x(n) \bigodot_{\alpha} h(n) \stackrel{F R F T}{\longleftarrow} \sqrt{2 \pi} X^{\alpha}(k) H(k \operatorname{cosec} \alpha)
$$

The Eq.(22) indicates the time domain fractional convolution and Eq.(23) relates the wavelet transform and the classical convolution process with an order $\alpha$ and this will be written in terms of $x(n)$ as,

$$
\begin{gathered}
W_{x}^{\alpha}(a, b)=x(n) \bigodot_{\alpha} \psi_{a, b}(n) \\
W_{x}^{\alpha}(a, b)=x(n) \odot \alpha\left\{\frac{1}{\sqrt{a}} \psi\left(\frac{t-b}{a}\right)\right\} \\
W_{x}^{\alpha}(a, b)=e^{-\left(\frac{j}{2}\right) b^{2} \cot \alpha}\left\{x(n) e^{-\left(\frac{j}{2}\right)^{2} n^{2} \cot \alpha} \psi_{a, b}(n)\right\} \\
W_{x}^{\alpha}(a, b)=\int_{-\infty}^{\infty} x(n) \psi_{\alpha, a, b}^{*} d n
\end{gathered}
$$

where, $\psi_{\alpha, a, b}^{*}(n)$ is the kernel function of the fractional wavelet transform which satisfies the following expression

$$
\psi_{\alpha, a, b}(n)=e^{-\left(\frac{j}{2}\right)\left(n^{2}-b^{2}\right) \cot \alpha} \psi_{a, b}(n)
$$

The inverse FRFT that FRWT is expressed in terms of FRFT $X^{\alpha}(k)$ of the signal $x(n)$ as,

$$
W_{x}^{\alpha}(a, b)=\int_{-\infty}^{\infty} \sqrt{2 \pi a} X^{\alpha}(k) \Psi^{*}(a k \operatorname{cosec} \alpha) K^{-\alpha} d \alpha
$$


where, $\Psi^{*}(a k \operatorname{cosec} \alpha)$ represents the Fourier transform of $\psi(n)$, the Eq.(29) shows that every fractional wavelet parameter scaled with fractional filter.

The FRWT expressed in Eq.(27) can be rewritten as,

$$
W_{x}^{\alpha}(a, b)=e^{-\left(\frac{j}{2}\right) b^{2} \cot \alpha} \int_{-\infty}^{\infty} x(n) e^{-\left(\frac{j}{2}\right)^{2} n^{2} \cot \alpha} \psi_{a, b}^{*}(n) d n
$$

\subsection{DISCRETE FRACTIONAL WAVELET TRANSFORM}

It is important that, FRWT should be converted into digital form and for this the mother wavelet function $\psi_{a, b}(n)$ is first discretized. Before discretizing, let us rearrange the expression for FRWT as,

$$
W^{\alpha}(a, b)=\int_{-\infty}^{\infty} x(n) \psi_{a, b}(n) d n
$$

where,

$$
x(n)=\int_{-\infty}^{\infty} x(k) K^{\alpha}(k, n) d k
$$

For optimum discretization of the mother wavelet, the popular method is $a=2^{m}$ and $b=n 2^{m}$, where $m$ and $n$ are the integers, and the discretized mother wavelet function can be written mathematically as,

$$
W_{a, b}=\frac{1}{\sqrt{2^{m}}} \psi\left(\frac{t-n 2^{m}}{2^{m}}\right)
$$

If the dilation parameter is fixed to 2 and localization parameter kept greater than zero, the fractional wavelet transform reduces to

$$
W_{a, b}^{\alpha}=\int_{-\infty}^{\infty} x(n) \frac{1}{\sqrt{2^{m}}} \psi\left(\frac{t-n 2^{m}}{2^{m}}\right) d n
$$

The Eq.(34) is the inner product between signal $X$ and $\psi_{a, b}$,

$$
\text { i.e. } W_{a, b}^{\alpha}=X \cdot \psi_{a, b}
$$

where, $W_{a, b}^{\alpha}$ is the $\alpha^{\text {th }}$ order DFRWT coefficient and is given by $(m, n)$.

For DFRWT, the coefficient $W_{a, b}^{\alpha}$ is called fractional wavelet coefficient. Now forward fractional wavelet transform is expressed as,

$$
\begin{gathered}
W_{m, n}^{\alpha}=\int_{-\infty}^{\infty} x(n) \frac{1}{\sqrt{2^{m}}} \psi\left(\frac{t-n 2^{m}}{2^{m}}\right) d n \\
W_{m, n}^{\alpha}=\int_{-\infty}^{\infty} x(n) \psi_{a, b}(n) d n
\end{gathered}
$$

Therefore, the IDFRWT can be defined as,

$$
x(n)=\sum_{a=-\infty}^{\infty} \sum_{b=-\infty}^{\infty} W_{a, b}^{\alpha} \psi_{a, b}
$$

By considering the scaling factor $\phi$, we get the approximation coefficient,

$$
\phi_{a, b}(n)=\frac{1}{\sqrt{2^{m}}} \phi\left(\frac{t-n 2^{m}}{2^{m}}\right)
$$

Satisfying $\int_{-\infty}^{\infty} \phi(n) d n=1$. where, $\phi_{0,0}(n) \approx \phi(n)$ and sometimes it is denoted as father wavelet.

The scaling function $\phi$ is multiplied in frequency domain to provide the complementary function $S_{a, b}^{\alpha}$ term as approximated coefficient related to DFRWT which can be expressed as,

$$
S_{a, b}^{\alpha}=\int_{-\infty}^{\infty} x(n) \phi_{a, b}(n) d n
$$

The weighted averages of approximated coefficients are nothing but continuous factor by $1 / \sqrt{2^{m}}$. To obtain the correct decompression, this results to,

$$
\begin{gathered}
x(n)=\sum_{b=-\infty}^{\infty} S_{a_{0}, b}^{\alpha} \phi_{a_{0}, b}(n)+\sum_{a=-\infty}^{\infty} \sum_{b=-\infty}^{\infty} W_{a, b}^{\alpha} \psi_{a, b}(n) \\
=X_{a_{0}}(n)+\sum_{a=-\infty}^{a_{0}} d_{a}(n)
\end{gathered}
$$

The Eq.(41), will be iteratively reduced to,

$$
x_{a-1}(n)=x_{a}(n)+d_{a}(n)
$$

The DFRWT is obtained by combining Fractional Fourier Transform (FRFT) and conventional wavelet transform.

\subsection{COMPUTING FFWT COMBINED WITH SPIHT}

Fast Fractional Wavelet Transform (FFRWT) is a combination of wavelet transform and FRFT. In the proposed method, the filter bank adopted is as per the JPEG2000 standards.

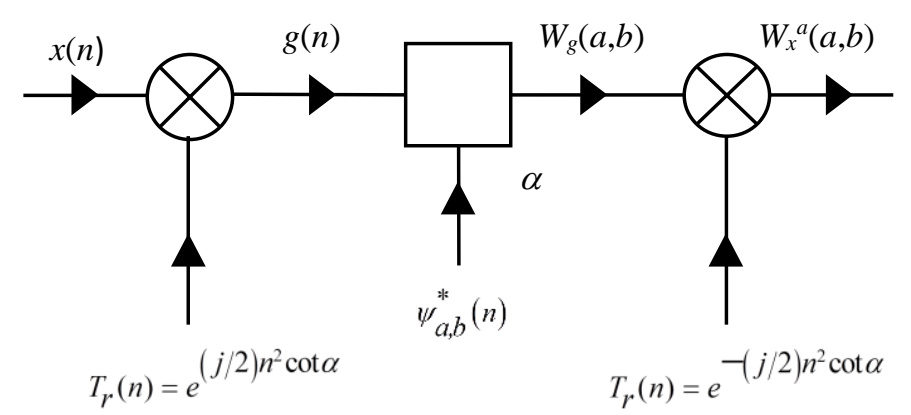

Fig.1. Computation efficient Fast Fractional Wavelet Transform

The Fig. 1 shows the internal structure of the FRWT. Since the input samples are discrete, and samples are obtained by a digital computing technique, it is essential to understand the discrete scheme. Firstly, the input function $x(n)$ is multiplied with the Chirpz signal, $e^{(j / 2) n^{2} \cot \alpha}$ and subsamples obtained as $g(n)$. Similarly, $a, b$ designated as scaling and translation parameters and are discretized. It is known that, very efficient method to discretize the scaling and translation parameter is by adopting the criteria $a=2^{m}$ and $b=n 2^{m}$, where, $m$ and $n$ are the control factor of integers and the localization parameter, which gives $W_{g}(a, b)$ Next, perform discrete wavelet transform with $W_{g}(a, b)$ on the samples of $g(n)$ taking the filter bank as 5/3, which leads to lossless image. Finally, obtain the product again with Chirps signal $e^{-(j / 2) b^{2} \cot \alpha}$, to get the Fast Fractional Wavelet Transform of an original signal $x(n)$ Although mathematically the process is complex, this can be easily implemented for practical applications. The complete overview of the proposed method 
adopting the Fast Fractional Wavelet transform is illustrated in Fig.2 and Fig.3 illustrates the structural flow of the proposed model.

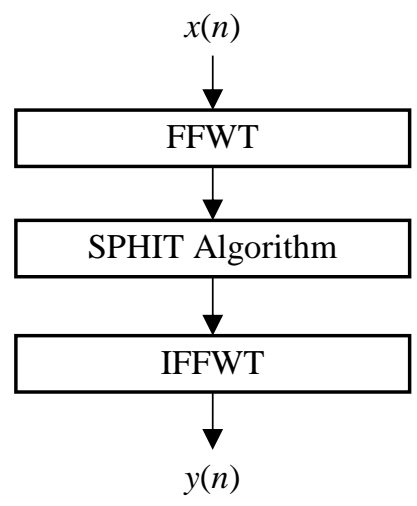

Fig.2. Block diagram of the proposed method

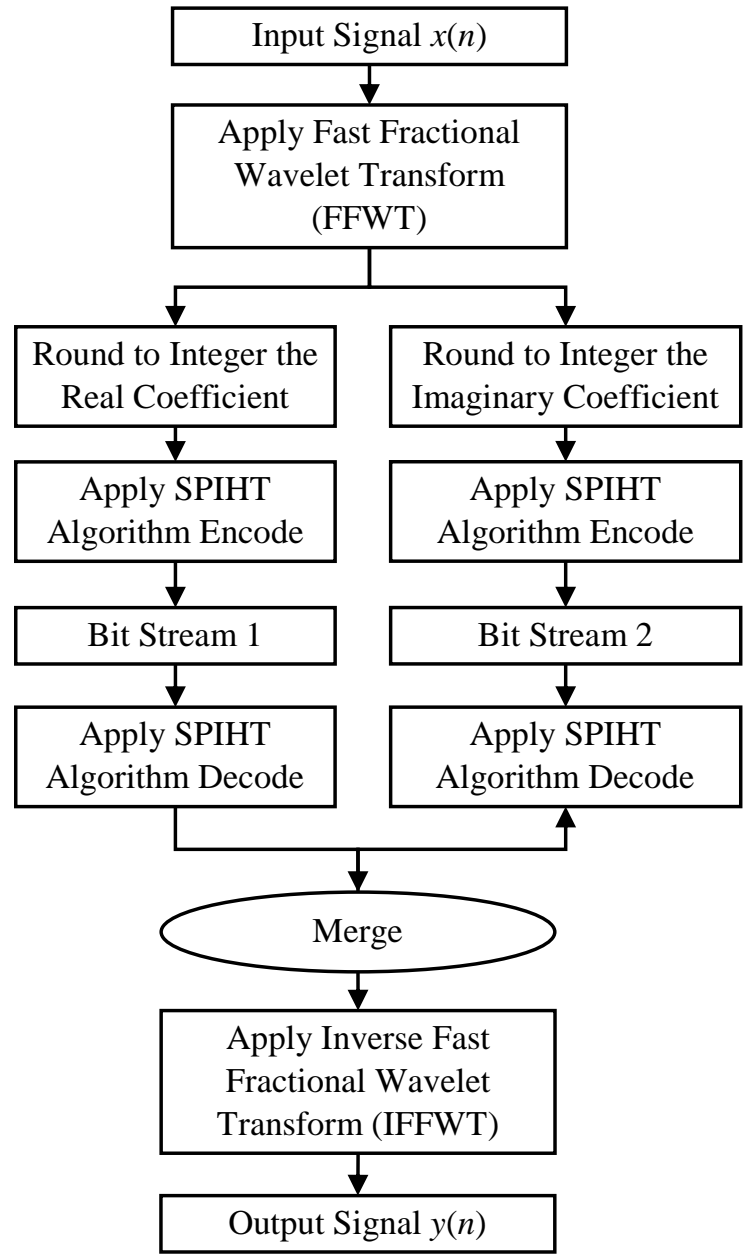

Fig.3. Structural Flow of the proposed Fast Fractional Wavelet Transform and SPIHT

The following procedure gives a brief outline of the proposed work.

- Multiply the original signal $x(n)$ with Chirpz signal in the time domain $x(n) \rightarrow g(n)$,

where, $g(n)=x(n) \cdot T_{r}(n)$ and $T_{r}(n)=e^{\left(\frac{j}{2}\right)^{2} \cot \alpha}$
- Apply wavelet transform to the signal $g(n)$

$$
g(n) \rightarrow W_{g}(a, b),
$$

where, $W_{g}(a, b)=g(n) * \psi_{a, b}^{*}(n)$

Multiply $W_{g}(a, b)$ with the Chirpz signal in transform domain

$$
W_{g}(a, b) \rightarrow W_{x}^{\alpha}(a, b)
$$

where,

$W_{x}^{\alpha}(a, b)=W_{g}(a, b) \cdot T_{r}(n)$ and $W_{x}^{\alpha}(a, b)=W_{g}(a, b) \cdot e^{-(j / 2) b^{2} \cot \alpha}$

- Apply Discrete Fractional Wavelet transform to the SPIHT encoder to get the bit stream.

- Feed the encoded bit stream to the SPIHT decoder.

- Apply the Inverse Discrete Fractional Fourier Transform to get back the original image $x(n)$.

- The chosen filter bank is biorthogonal filter bank.

- The BFB 9/3 Filter bank has better PSNR, correlation coefficient and MSE compared to other filter banks.

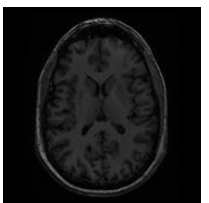

(a) $2 / 2$ filter bank

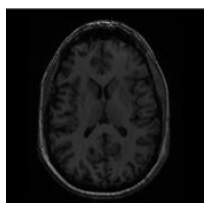

(e) $9 / 3$ filter bank

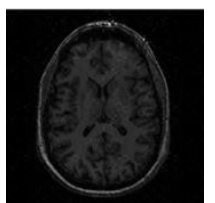

(i) $8 / 4$ filter bank

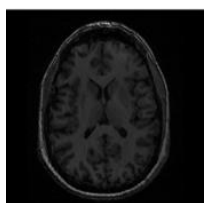

(m) $9 / 7$ filter bank

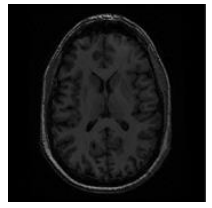

(b) $6 / 2$ filter bank

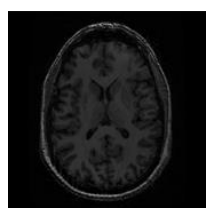

(f) $13 / 3$ filter bank

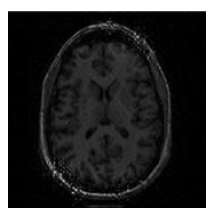

(j) 12/4 filter bank

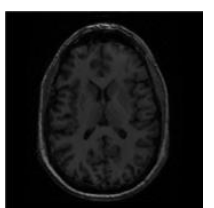

(n) $9 / 11$ filter (o) $17 / 11$ filter bank

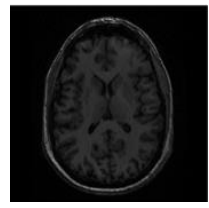

(c) 10/2 filter bank

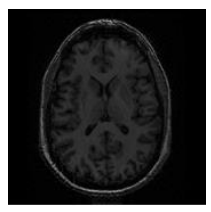

(g) $17 / 3$ filter bank

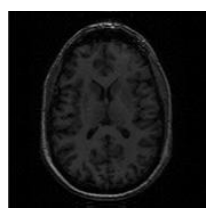

(k) $16 / 4$ filter bank

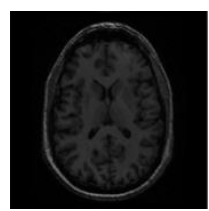

bank

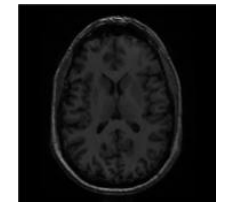

(d) $5 / 3$ filter bank

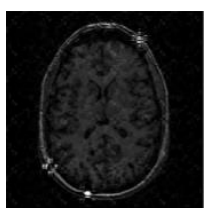

(h) 4/4 filter bank

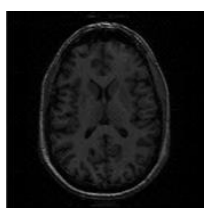

(1) $20 / 4$ filter bank
Fig.4. Compressed brain image with various biorthogonal filter banks 


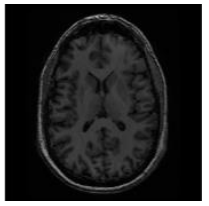

(a) $2 / 2$ filter bank

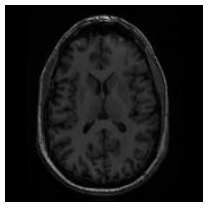

(e) $9 / 3$ filter bank

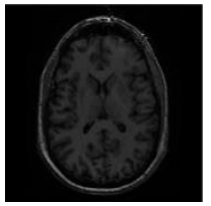

(i) $8 / 4$ filter bank

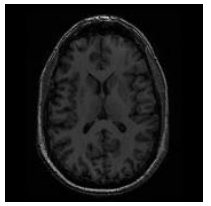

(m) $9 / 7$ filter bank

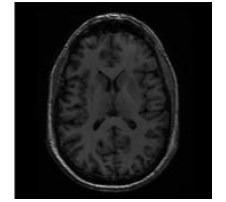

(b) $6 / 2$ filter bank

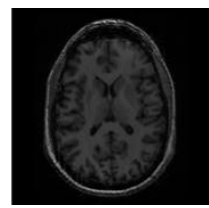

(f) $13 / 3$ filter bank

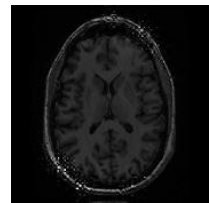

(j) 12/4 filter bank

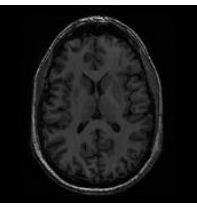
bank (n) $9 / 11$ filter

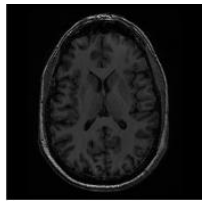

(c) $10 / 2$ filter bank

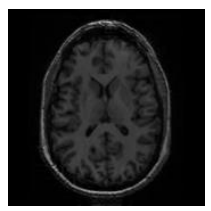

(g) 17/3 filter bank

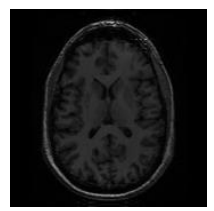

(k) $16 / 4$ filter bank

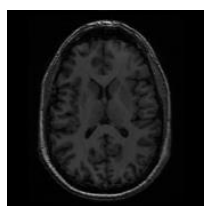

bank (o) $17 / 11$ filter

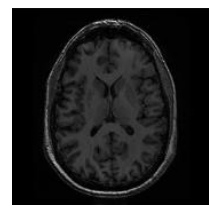

(d) $5 / 3$ filter bank

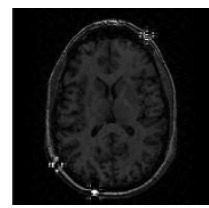

(h) 4/4 filter bank

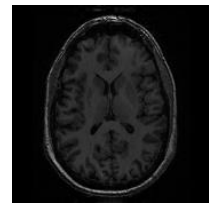

(1) 20/4 filter bank
Fig.5. Decompressed brain images with various Bi-orthogonal filter banks

Table.1. Subjective Quality Metrics for Various Biorthogonal Filter Banks of Brain Images for $\alpha=0.91$

\begin{tabular}{|c|c|c|c|c|c|}
\hline $\begin{array}{c}\text { Filter } \\
\text { Bank }\end{array}$ & BPP $^{*}$ & CR $^{*}$ & PSNR $^{*}$ & CC$^{*}$ & MSE \\
\hline $2 / 2$ & 0.595272 & 5.014819 & 38.114915 & 0.990686 & 0.901443 \\
\hline $6 / 2$ & 0.724743 & 4.638373 & 43.575863 & 0.999016 & 0.905334 \\
\hline $10 / 2$ & 0.746166 & 4.581465 & 41.816692 & 0.998081 & 1.263763 \\
\hline $5 / 3$ & 0.709087 & 4.680861 & 43.901157 & 0.998805 & 1.036697 \\
\hline $9 / 3$ & 0.712421 & 4.671747 & 44.535501 & 0.999021 & 0.772797 \\
\hline $13 / 3$ & 0.616394 & 4.949288 & 37.985670 & 0.990781 & 3.544785 \\
\hline $17 / 3$ & 0.641392 & 4.873913 & 36.957780 & 0.988179 & 3.574188 \\
\hline $4 / 4$ & 0.904080 & 3.802136 & 26.107889 & 0.892075 & 13.10260 \\
\hline $8 / 4$ & 0.919781 & 4.167143 & 33.968086 & 0.985344 & 4.429855 \\
\hline $12 / 4$ & 0.813717 & 4.410832 & 27.840419 & 0.908239 & 7.045502 \\
\hline $16 / 4$ & 0.940342 & 4.122985 & 34.880892 & 0.986217 & 3.329666 \\
\hline $20 / 4$ & 0.959034 & 4.083645 & 36.721642 & 0.993748 & 3.349854 \\
\hline $9 / 7$ & 0.656044 & 4.830790 & 42.404882 & 0.997080 & 1.686188 \\
\hline $9 / 11$ & 0.925201 & 4.155409 & 43.480792 & 0.998528 & 1.061035 \\
\hline $17 / 11$ & 0.652565 & 4.840959 & 42.258411 & 0.997085 & 1.558533 \\
\hline
\end{tabular}

Note: $\mathbf{B P P}^{*}=\mathrm{BPP}=\mathrm{Bits}$ per Pixel, $\mathbf{C R}^{*}=$ Compression Ratio, PSNR $^{*}=$ Peak to Signal Noise Ratio, $\mathbf{C C}^{*}=$ Correlation, Coefficient and MSE $^{*}=$ Mean Square Error.

\section{EXPERIMENTAL RESULTS}

The combination of FFWT and SPIHT approach was experimented with Matlab environment. To validate the proposed technique, images of brain having the size $512 \times 512$ were considered as experimental datasets. The performance of the proposed model was evaluated with five performance measures as mentioned in Table.1. These parameters were experimented with the different Biorthogonal Filter Banks (BFB) with $\alpha=0.91$. The results are tabulated in Table.1. Similarly, Fig.4 and Fig.5 describes the compressed and decompressed image of brain using various filter banks. The results were compared with the existing techniques [22], [23].

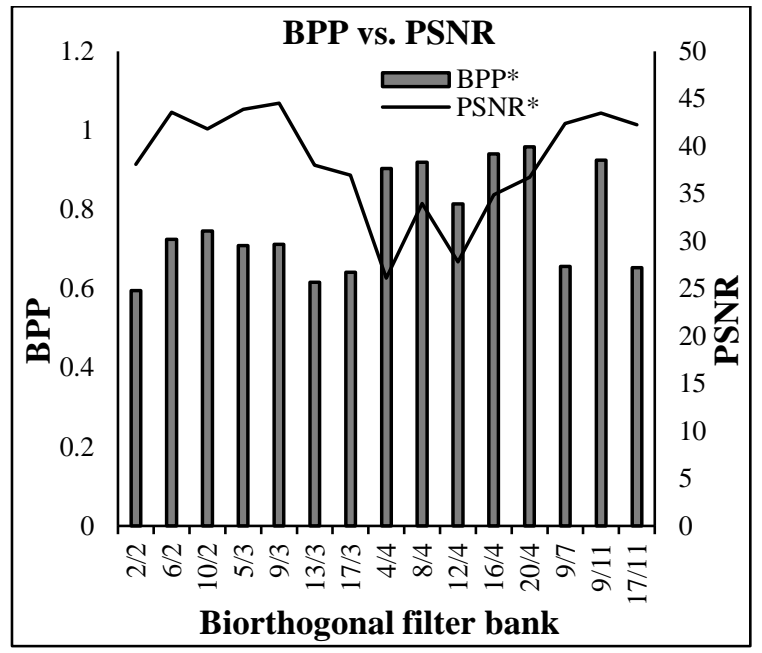

Fig.6. Relationship between BPP and PSNR for various biorthogonal filters banks

The Fig.6, Fig.7 and Fig.8 illustrate the different filter banks with parameters of PSNR, bits per pixel, compression ratio and MSE.

The Fig.6 shows the variation in bits per pixel versus peak to signal noise ratio. It is observed that BFB $9 / 3$ provides better PSNR resulting good quality of compressed image as compared to the existing filter banks with the bits per pixel of 0.712 . BFB $5 / 3$ has fewer bits per pixel than BFB 9/3 with less PSNR. The BFB 12/4 has the least PNSR which yield the worst quality of the compressed image, and gives more bits per pixel. The highest bit per pixel is BFB 9/11 with PSNR 43.48, and the least bits per pixel are BFB $2 / 2$ with moderate PSNR.

The Fig.7 shows the relation between compression ratio achieved and PSNR. The compression ratio is high in BFB 2/2 compared to other filter bank with moderate PSNR. The BFB 9/3 provides a good compressed image quality with a PSNR of 44.53 and a compression ratio of 4.41 , the BFB $17 / 11$ has better PSNR and compression ratio as compared to another filter bank. The filter bank BFB 4/4 does not give good quality of compressed image with the compression of 3.80 and PSNR of 26.10. 


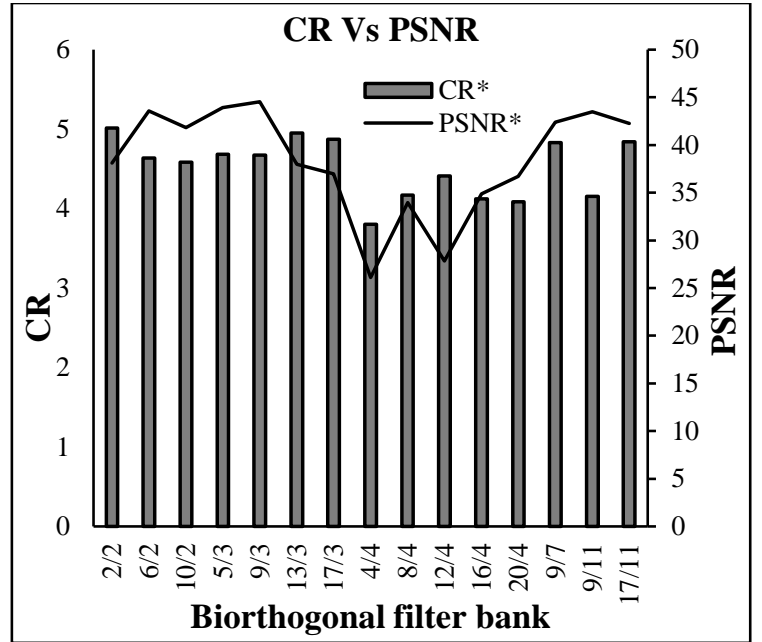

Fig.7. Relationship between Compression Ratio and PSNR for various biorthogonal filters banks

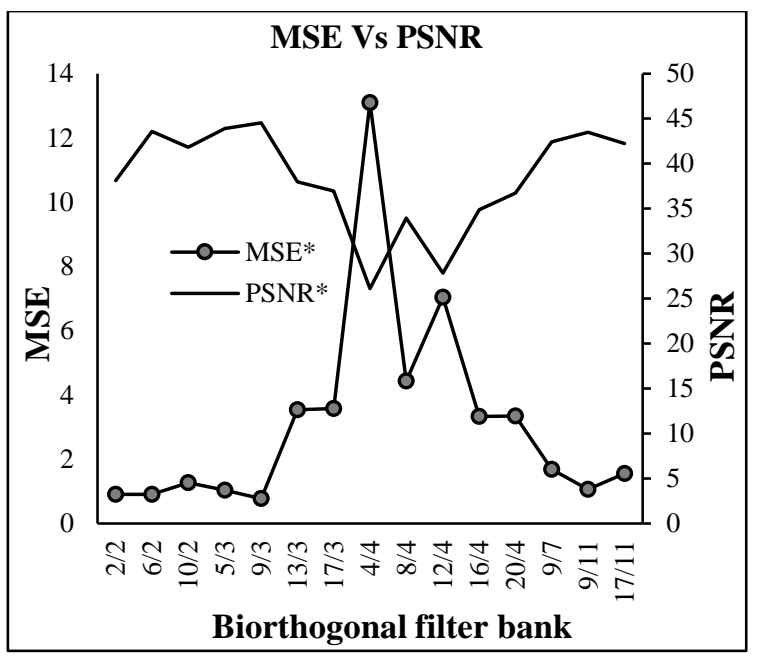

Fig.8. Relationship between Compression MSE and PSNR for various biorthogonal filter banks

The Fig. 8 shows the relationship between the MSE and PSNR. It is observed that, there is a tradeoff between MSE and PSNR That means as PSNR increases, MSE will decrease and vice versa. Higher PNSR yield less error and low PSNR yields serious error. The BFB 9/3 has less error with the PSNR of 44.53, and the BFB 9/11 has moderate PNSR with average error. The BFB 12/4 has serious error with the PSNR of 27.84 .

\section{CONCLUSION}

In this paper, the performance analysis of image compression based on FFWT combined with SPIHT was proposed. This method has explicit physical interpretation and verified the necessity of FRWT and SPIHT technique. Some fundamental results of this transform were presented including PSNR, Correlation coefficient, Compression ratio and admissibility condition. This transform considers merits of the localization which is present in the FRFT to improve performance in decompression wavelet transform. It is known fact that, the FRWT is good in presenting information simultaneously in time and fractional domain and also, presented information in the time fractional- frequency plane. Thus, this method will overcome the drawbacks of the wavelet transform and the FRFT. The proposed technique is very simple to implement and can be applied to any real time problems and less computational complexity.

\section{REFERENCES}

[1] Stephane G. Mallat, "A Theory for Multiresolution Signal Decomposition: The Wavelet Representation", IEEE Transactions on Pattern Analysis and Machine Intelligence, Vol. 11, No. 7, pp. 674-693, 1989.

[2] Yetik I. Samil, "Image Representation and Compression with the Fractional Fourier Transform", Optics Communications, Vol. 197, No. 4-6, pp. 275-278, 2001.

[3] R.M. Rao and A.S. Bopardikar, "Wavelet Transform: Introduction to Theory and Applications", Prentice Hall, 1998.

[4] B.E. Usevitch, "A Tutorial on Modern Lossy Wavelet Image Compression: Foundations of JPEG 2000", IEEE Signal Processing Magazine, Vol. 18, No. 5, pp. 22-35, 2001.

[5] V.K. Goyal, "Theoretical Foundations of Transform Coding”, IEEE Signal Processing Magazine, Vol. 18, No. 5, pp. 9-21, 2001.

[6] H.M. Ozaktas, Z. Zalevsky and M.A. Kutay, "The Fractional Fourier Transform with Applications in Optics and Signal Processing", John Wiley, 2001.

[7] Ying Huang, "The Fractional Wave Packet Transform", Multidimensional Systems and Signal Processing, Vol. 9, No. 4, pp. 399-402, 1998.

[8] E. Sejdic, I. Djurovic and L. Stankovic, "Fractional Fourier Transform as a Signal Processing Tool: An Overview of Recent Developments", Signal Processing, Vol. 91, No. 6, pp. 1351-1369, 2011.

[9] A.K Singh and R. Saxena, "DFRFT: A Classified Review of Recent Methods with its Application", Journal of Engineering, Vol. 2013, No. 1, pp. 1-13, 2013.

[10] G. Bhatnagar, Q.M. Jonathan Wu and B. Raman, "Discrete Fractional Wavelet Transform and its Application to Multiple Encryption”, Information Sciences, Vol. 223, pp. 297-316, 2013.

[11] Ran Tao, Yan-Lei Li and Yue Wang, "Short-Time Fractional Fourier Transform and it's Applications", IEEE Transactions on Signal Processing, Vol. 58, No. 5, pp. 2568-2580, 2010.

[12] Amir Said and W.A. Pearlman, "A New, Fast and Efficient Image Code based on Set Partitioning in Hierarchical Trees", IEEE Transactions on Circuits Systems Video Technology, Vol. 6, No. 3, pp. 1-16, 1996.

[13] C. Vijaya and J.S. Bhat, "Signal Compression using Discrete Fractional Fourier Transform and Set Partitioning in Hierarchical Tree", Signal Processing, Vol. 86, No. 8, pp. 1976-1983, 2006.

[14] S. Pushpa Mala, D. Jayadevappa and K. Ezhilarasan, "Application of Fractional Wave Packet Transform for Robust Watermarking of Mammograms", International Journal of Telemedicine and Applications, Vol. 2015, pp. 18, 2015.

[15] S.C. Pei and M.H. Yeh, "A Novel Method for Discrete Fractional Fourier Transform Computation”, Proceedings of 
IEEE International Symposium on Circuits and Systems, pp. 587-588, 2001.

[16] V.A. Narayanan and K.M.M. Prabhu, "The Fractional Fourier Transform: Theory, Implementation and Error Analysis", Microprocessor and Microsystems, Vol. 27, No. 10, pp. 511-521, 2003.

[17] Wen-Liang Hsue and Soo-Chang Pei, "Rational-Ordered Discrete Fractional Fourier Transform", Proceedings of $20^{\text {th }}$ European Conference on Signal Processing, pp. 2124-2127, 2012.

[18] M.H. Yeh and S.C. Pei, "A Method for the Discrete Fractional Fourier Transform Computation", IEEE Transactions on Signal Processing, Vol. 51, No. 3, pp. 889891, 2003.

[19] C. Candan, M.A. Kutay and H.M. Ozaktas, "The Discrete Fractional Fourier Transform", IEEE Transactions on Signal Processing, Vol. 48, No. 5, pp. 1329-1337, 2000.
[20] M.H. Yeh and S.C. Pei, "A Method for the Discrete-Time Fractional Fourier Transform Computation", IEEE Transactions on Signal Processing, Vol. 51, No. 3, pp. 889891, 2003.

[21] M. H. Yeh, "Angular Decompositions for the Discrete Fractional Signal Transforms", Signal Processing, Vol. 85, No. 3, pp. 537-547, 2005.

[22] M.A. Raposo-Sanchez, J. Saez-Landete and F. Cruz-Roldan, " $\alpha$-Spline Design of Finite Impulse Response Digital Filters”, Signal Processing, Vol. 122, pp. 204-212, 2016.

[23] Banu Unalm Uzun, "On the Fractional Fourier and Continuous Fractional Wave Packet Transforms of Almost Periodic Functions", Journal of Inequalities and Applications, Vol. 126, No. 1, pp. 126-130, 2017. 